\title{
Epistaxis during Inhalation Sedation Using Nitrous Oxide: A Rare Case Report
}

\author{
Shivani Mathur ${ }^{1}$, Tushar Pruthi ${ }^{2}$, Vinod Sachdev ${ }^{3}$, Rahul Chopra ${ }^{4}$, Sakshi Jain ${ }^{5}$
}

\begin{abstract}
Nitrous oxide is widely being used as a behavior management technique to reduce the anxiety of the patient by pediatric dentists worldwide. Nitrous oxide inhalational sedation (NOIS) exhibits a remarkable safety profile with rarely reported cases of complications associated with it. This case report highlights the complication of NOIS of an 8-year-old boy. On administration of nitrous oxide, nasal bleeding occurred. Nasal bleeding was controlled immediately and planned treatment was aborted. This case report explored various possibilities that can lead to epistaxis in a young child. But the question remains whether nitrous oxide triggered nasal bleed or did it happen due to any other local or systemic cause?
\end{abstract}

Keywords: Complications, Epistaxis, Nitrous oxide.

Journal of South Asian Association of Pediatric Dentistry (2020): 10.5005/jp-journals-10077-3060

\section{INTRODUCTION}

Dental visit induces fear and anxiety which increases stress level and agony in the patient as well as the dentist. Though different nonpharmacological methods are present to manage dental fear and anxiety, severe anxiety might not be effectively managed by these methods. Therefore, treatment of such patients requires pharmacological management, such as, general anesthesia and sedation. Children are not able to repress their fear, thereby resulting in excessive crying and physical struggle. A survey conducted by the members of the International Society of Pediatric Dentistry (IAPD) and the European Academy of Pediatric Dentistry (EAPD) reported $46 \%$ of pediatric dentists to use nitrous oxide inhalation sedation as a technique to achieve behavior modification in Europe followed by Asia and North America. ${ }^{1,2}$ Various countries which comprise the US, Australia, and France use nitrous oxide in numerous departments, such as, dental, radiology, orthopedics, and emergencies. ${ }^{3-6}$ Though nitrous oxide inhalation sedation is considered to be very safe yet few instances of various complications have been documented in the literature. This case report highlights the incidence of epistaxis in a child indicated for extraction under nitrous oxide inhalation sedation at a concentration of $40 \% \mathrm{~N}_{2} \mathrm{O}$.

\section{Case Description}

An 8-year-old male patient reported to the Outpatient Department of Pediatric and Preventive Dentistry with pain in the right lower back tooth region for 3 days. The pain was sharpshooting and intermittent in nature which aggravated while eating and subsided only after medication. On examination, 84 was grossly decayed and was indicated for extraction. No relevant past medical history was reported by the patient. The child showed a Frankl rating of 2 and extraction was planned to be executed under nitrous oxide inhalation sedation. Informed consent was taken from the parents before executing nitrous oxide sedation and the parents were informed of the pharmacological drug along with postoperative instructions. Inhalation was executed as per the protocol and while the patient reached a level of $40 \% \mathrm{~N}_{2} \mathrm{O}$, it started bleeding from \begin{tabular}{l} 
1,3,5 Department of Pediatric and Preventive Dentistry, ITS-CDSR, \\
Ghaziabad, Uttar Pradesh, India \\
2Department of Pedodontics and Preventive Dentistry, Subharti \\
Dental College, Swami Vivekanand Subharti University, Meerut, Uttar \\
Pradesh, India \\
${ }^{4}$ Department of Periodontology, ITS-CDSR, Ghaziabad, Uttar Pradesh, \\
India \\
Corresponding Author: Shivani Mathur, Department of Pediatric \\
and Preventive Dentistry, ITS-CDSR, Ghaziabad, Uttar Pradesh, India, \\
Phone: +91 8826221676, e-mail: shivanimathur.dr@gmail.com \\
How to cite this article: Mathur S, Pruthi T, Sachdev V, et al. Epistaxis \\
during Inhalation Sedation Using Nitrous Oxide: A Rare Case Report. \\
J South Asian Assoc Pediatr Dent 2020;3(2):92-94. \\
Source of support: Nil \\
Conflict of interest: None \\
\hline \hline
\end{tabular}

the nose. The patient was immediately given oxygen flush and the mask was removed. Continuous pressure for about 5-7 minutes was applied to the nose by pinching the tip of the nose between the thumb and forefinger. The nose was re-examined for any fresh bleeding and the procedure was aborted for the day under conscious sedation, though the extraction was performed after half an hour of the incidence. The patient was discharged after assuring for a cessation of nasal bleed.

Nitrous oxide takes 2-3 minutes for its onset and recovery due to its rapid absorption. ${ }^{7}$ The cough reflex is guarded since there is negligible damage to any of the reflexes. ${ }^{8,9}$ Nitrous oxide inhalational sedation (NOIS) rarely causes silent regurgitation and aspiration which can cause a problem with pharyngeal-laryngeal reflexes. However, this can be circumvented by maintaining the consciousness of the patient. ${ }^{10}$ However, till now there is no documented mortality or any cases of serious complications when nitrous oxide is administered within the suggested concentration, thus exhibiting a remarkable safety profile. ${ }^{11,12}$

Various studies have been conducted on the safety and efficacy of nitrous oxide as a pharmacological sedation agent. ${ }^{6,7}$ The most common adverse effect which was found to be associated with

(c) The Author(s). 2020 Open Access This article is distributed under the terms of the Creative Commons Attribution 4.0 International License (https://creativecommons. org/licenses/by-nc/4.0/), which permits unrestricted use, distribution, and non-commercial reproduction in any medium, provided you give appropriate credit to the original author(s) and the source, provide a link to the Creative Commons license, and indicate if changes were made. The Creative Commons Public Domain Dedication waiver (http://creativecommons.org/publicdomain/zero/1.0/) applies to the data made available in this article, unless otherwise stated. 
$\mathrm{N}_{2} \mathrm{O}$ is vomiting. It has been recorded that a lengthy procedure or a deeper level of sedation increases the prevalence of minor adverse events. $^{2}$

Another adverse event is diffusion hypoxia that occurs after the termination of nitrous oxide that can be decreased by supplying $100 \%$ oxygen as soon as nitrous oxide is discontinued..$^{2,13}$ Recently, a case of laryngospasm was documented with NOIS at a higher (70\%) concentration of $\mathrm{N}_{2} \mathrm{O}$. This could be because $70 \%$ of $\mathrm{N}_{2} \mathrm{O}$ may lead some patients into deep sedation. 2,14

Various other adverse events, i.e., at $\mathrm{N}_{2} \mathrm{O}$ concentration at $70 \%$ stabbing pain in the chest, stridor, tonic-clonic seizure, and oxygen desaturation have been reported while apnea for $>15$ seconds has been observed in relation to $50 \%$ concentration of $\mathrm{N}_{2} \mathrm{O} .2 \mathrm{~N}_{2} \mathrm{O}$ has also been reported to cause irreversible inactivation of vitamin B12 which may further result in megaloblastic anemia. ${ }^{2,15}$ In a 311 member survey of IAPD and EAPD, one case of death with $\mathrm{N}_{2} \mathrm{O}$ has been reported though specific details are lacking., ${ }^{1,2}$

In a nondental setting, a very common finding in the age group of 2-10 years is the nasal bleed which is known as epistaxis. Epistaxis occurs due to disruption or irritation in the lining of the nasal membrane leading to abnormal bleeding. The inner nasal membrane that is rich in blood vessels and lies close to the surface, is more prone to injury. Ethmoid branches of the internal carotid arteries and the facial and internal maxillary divisions of the external carotid arteries supply this region. ${ }^{16}$

While bleeding from the anterior part of the nose is mainly due to the presence of a lot of blood vessels, i.e., Kiesselbach's plexus, also known as Little's area. The back of the nose bleed is mainly due to Woodruff's plexus and is known as a posterior bleed. The posterior bleed is usually with bleeding from both nostrils and is difficult to control resulting in a higher risk of airway owing to a greater flow of blood into the posterior pharynx. ${ }^{16}$

Epistaxis may result due to various local, systemic, environmental, and medication-induced factors. Local causes include digital manipulation, a deviated septum, trauma, inhaled corticosteroids, and chronic nasal cannula use while systemic causes constitute alcoholism, hypertension, vascular malformations, or coagulopathies (von Willebrand disease, hemophilia). Environmental factors usually include allergies or dryness during the winter months. Various medications include nonsteroidal antiinflammatory drugs (NSAIDS) (ibuprofen), anticoagulants (warfarin), platelet aggregation inhibitors (clopidogrel), or supplement/ alternative medications and some herbs like garlic, ginseng, and ginkgo. ${ }^{17}$ Furthermore, history regarding any nasal blockage, facial pain, headaches, facial deformity, or drug abuse (cocaine) needs to be recorded while taking a history of the patient.

In the present case, the patient did not exhibit any systemic illness, thus, epistaxis could be due to irritation owing to nitrous oxide use or thin nasal lining or previous injury to the nose. Also, the nasal bleed was easily controlled in the present case. However, if there is continuous bleeding even after applying pressure, then a thorough investigation should be conducted by an otolaryngologist which usually includes the application of hemostatic agents (oxymetazoline or dilute phenylephrine/ epinephrine), anterior nasal packing, and/or chemical or electrical cautery. Rarely, posterior nasal packing or arterial embolization may be required. It is reported that most episodes of epistaxis occur during the winter and in dry climates. ${ }^{18}$ New and repeated incidents of epistaxis can be avoided by keeping the nasal mucosa moist with the help of saline nasal spray, petroleum jelly, or humidifier. ${ }^{19}$

The review of the literature shows no reported case of epistaxis during NOIS to the best of our knowledge. However, this nasal bleed was easily controlled and the patient was successfully treated without any complication. This paper intends to highlight a very rare, probable complication of NOIS, but as a caregiver, it becomes our responsibility not to panic in such a situation and control epistaxis by simple measures like pressure packs.

\section{Conclusion}

To the best of the author's knowledge, this is the first reported incidence of epistaxis during NOIS. However, the underlying cause could not be ascertained as epistaxis is a common finding in this age group. Authors also recommend recording the history of epistaxis as a part of regular history taking before the nitrous oxide sedation procedure.

\section{References}

1. Wilson S, Alcaino EA. Survey on sedation in pediatric dentistry: a global perspective. Int J Paediatr Dent 2011;21(5):321-332. DOI: 10.1111/j.1365-263X.2011.01127.x.

2. In Chi S. Complications caused by nirous oxide in dental sedation. J Dent Anesth Pain Med 2018;18(2):71-78. DOI: 10.17245/ jdapm.2018.18.2.71.

3. Pasarón R, Burnweit C, Zerpa J, et al. Nitrous oxide procedural sedation in non-fasting pediatric patients undergoing minor surgery: a 12-year experience with 1,058 patients. Pediatr Surg Int 2015;31(2):173-180. DOI: 10.1007/s00383-014-3608-5.

4. Tsze DS, Mallory MD, Cravero JP. Practice patterns and adverse events of nitrous oxide sedation and analgesia: a report from the pediatric sedation research consortium. J Pediatr 2016;169:260-265.e2. DOI: 10.1016/j.jpeds.2015.10.019.

5. Zier JL, Liu MX. Safety of high-concentration nitrous oxide by nasal mask for pediatric procedural sedation experience with 7802 cases. Pediatr Emerg Care 2011;27(12):1107-1112. DOI: 10.1097/ PEC.0b013e31823aff6d.

6. Onody P, Gil P, Hennequin M. Safety of inhalation of a $50 \%$ nitrous oxide/oxygen premix - a prospective survey of 35828 administrations. Drug Saf 2006;29(7):633-640. DOI: 10.2165/00002018-20062907000008.

7. Annequin D, Carbajal R, Chauvin $P$, et al. Fixed $50 \%$ nitrous oxide oxygen mixture for painful procedures: a french survey. Pediatrics 2000;105(4):e47. DOI: 10.1542/peds.105.4.e47Available at emedicine. medscape.com/article/863220-overview.

8. American Academy of Pediatric Dentistry (AAPD). Guideline on use of nitrous oxide for pediatric dental patients. Pediatr Dent 2013;35:200-204.

9. Paterson SA, Tahmassebi JF. Pediatric dentistry in the new millennium: use of inhalation sedation in pediatric dentistry. Dent Update 2003;30(7):350-356. DOI: 10.12968/denu.2003.30.7.350, 358.

10. Hogue $D$, Ternisky M, Iranour B. The response to nitrous oxide analgesia in children. ASDC J Dent Child 1971;38(2):129-133.

11. Holyroyd I. Conscious sedation in pediatric dentistry: a short review of the current UK guidelines and the technique of inhalational sedation with nitrous oxide. Paediatr Anaesth 2008;18(1):13-17. DOI: 10.1111/j.1460-9592.2007.02387.x.

12. Lyratzopoulos G, Blain KM. Inhalation sedation with nitrous oxide as an alternative to dental general anesthesia for children. J Public Health Med 2003;25(4):303-312. DOI: 10.1093/pubmed/fdg068.

13. Duncan $\mathrm{GH}$, Moore P. Nitrous-oxide and the dental patient - a review of adverse reactions. J Am Dent Assoc 1984;108(2):213-219. DOI: 10.14219/jada.archive.1984.0462. 
14. Babl FE, Grindlay J, Barrett MJ. Laryngospasm with apparent aspiration during sedation with nitrous oxide. Ann Emerg Med 2015;66(5):475-478. DOI: 10.1016/j.annemergmed.2015. 04.029 .

15. Amess JA, Burman JF, Rees GM, et al. Megaloblastic haemopoiesis in patients receiving nitrous oxide. Lancet 1978;2(8085):339-342. DOI: 10.1016/S0140-6736(78)92941-0.

16. Tabassom A. Julia J. Cho. Epistaxis (Nose Bleed). Treasure Island (FL): StatPearls Publishing; 2018.
17. Kelly P. Epistaxis and nasal foreign body removal Dehn RW, Asprey DP, ed. Essential Clinical Procedures. 3rd ed., Philadelphia, Pa: Elsevier Saunders; 2013. pp. 166-178.

18. Chandrakantan A, Mohammad S, Adler AC. Anesthetic management of acute nasal epistaxis occurring during general anesthesia. Paediatr Anaesth 2019;29(8):872-874. DOI: 10.1111/pan.13678.

19. Smith J, Hanson J, Chowdhury R, et al. Community-based management of epistaxis: who bloody knows? Can Pharm J (Ott) 2019;152(3):164-176. DOI: 10.1177/1715163519840380. 